\title{
Appraising the effectiveness of electrical and magnetic brain stimulation techniques in acute major depressive episodes: an umbrella review of meta-analyses of randomized controlled trials
}

\author{
Laís B. Razza, ${ }^{1}$ iD Leonardo Afonso dos Santos, ${ }^{1}$ Lucas Borrione, ${ }^{1}$ iD Helena Bellini, ${ }^{1,2}$ iD \\ Luis C. Branco, ${ }^{3}$ iD Eric Cretaz, ${ }^{4}$ Dante Duarte, ${ }^{3,5}$ Ygor Ferrão, ${ }^{6}$ Ricardo Galhardoni, ${ }^{7,8}$ João \\ Quevedo,,${ }^{9,10,11,12}$ iD Marcel Simis, ${ }^{13}$ Felipe Fregni, ${ }^{14}$ Christoph U. Correll, ${ }^{15,16,17}$ Frank Padberg, ${ }^{18}$ \\ Alisson Trevizol, ${ }^{19,20}$ Zafiris J. Daskalakis, ${ }^{20,21}$ Andre F. Carvalho, ${ }^{20,21}$ Marco Solmi, ${ }^{22}$ iD André R. \\ Brunoni $^{1,23,24,25}$ iD
}

\begin{abstract}
${ }^{1}$ Serviço Interdisciplinar de Neuromodulação, Laboratório de Neurociências (LIM27), Departamento e Instituto de Psiquiatria, Hospital das Clínicas da Faculdade de Medicina da Universidade de São Paulo (HCFMUSP), São Paulo, SP, Brazil. ${ }^{2}$ Laboratório de Neuroimagem em Psiquiatria (LIM21), Departamento e Instituto de Psiquiatria, HCFMUSP, São Paulo, SP, Brazil. ${ }^{3}$ Neuromodulation and Clinical Research Center, Spaulding Rehabilitation Hospital, Harvard Medical School, Boston, MA, USA. ${ }^{4}$ Serviço de Eletroconvulsoterapia, Departamento e Instituto de Psiquiatria, HCFMUSP, São Paulo, SP, Brazil. ${ }^{5}$ Department of Psychiatry and Behavioural Neurosciences, McMaster University, Hamilton, ON, Canada. ${ }^{6}$ Departamento de Clínica Médica, Universidade Federal de Ciências da Saúde de Porto Alegre (UFCSPA), Porto Alegre, RS, Brazil. ${ }^{7}$ Faculdade de Medicina, Universidade Cidade de São Paulo (UNICID), São Paulo, SP, Brazil. ${ }^{8}$ Centro de Dor (LIM-62), Departamento de Neurologia, HCFMUSP, São Paulo, SP, Brazil. ${ }^{9}$ Translational Psychiatry Program, Faillace Department of Psychiatry and Behavioral Sciences, McGovern Medical School, The University of Texas Health Science Center at Houston (UTHealth), Houston, TX, USA. ${ }^{10}$ Center of Excellence on Mood Disorders, Faillace Department of Psychiatry and Behavioral Sciences, UTHealth, McGovern Medical School, Houston, TX, USA.

${ }^{11}$ Neuroscience Graduate Program, The University of Texas, MD Anderson Cancer Center, UTHealth Graduate School of Biomedical Sciences, Houston, TX, USA. ${ }^{12}$ Laboratório de Psiquiatria Translacional, Programa de Pós-Graduação em Ciências da Saúde, Universidade do Extremo Sul Catarinense (UNESC), Criciúma, SC, Brazil. ${ }^{13}$ Instituto de Medicina Física e Reabilitação, HCFMUSP, São Paulo, SP, Brazil. ${ }^{14}$ Laboratory of Neuromodulation \& Center for Clinical Research Learning, Department of Physical Medicine and Rehabilitation, Spaulding Rehabilitation Hospital, Harvard Medical School, Boston, MA, USA. ${ }^{15}$ Department of Psychiatry, The Zucker Hillside Hospital, Northwell Health, Glen Oaks, NY, USA. ${ }^{16}$ Department of Psychiatry and Molecular Medicine, Donald and Barbara Zucker School of Medicine at Hofstra/Northwell, Hempstead, NY, USA.

${ }^{17}$ Department of Child and Adolescent Psychiatry, Charité Universitätsmedizin Berlin, Berlin, Germany. ${ }^{18}$ Department of Psychiatry and Psychotherapy, Ludwig Maximilian University Munich, Munich, Germany. ${ }^{19}$ Temerty Centre for Therapeutic Brain Intervention, Centre for Addiction and Mental Health, Toronto, ON, Canada. ${ }^{20}$ Department of Psychiatry, University of Toronto, Toronto, ON, Canada. ${ }^{21}$ Centre for Addiction and Mental Health (CAMH), Toronto, ON, Canada. ${ }^{22}$ Dipartimento di Neuroscienze, Università Degli Studi Di Padova, Padova, Italy. ${ }^{23} \mathrm{HCFMUSP,} \mathrm{São}$ Paulo, SP, Brazil. ${ }^{24}$ Laboratório de Neurociências (LIM27), Departamento e Instituto de Psiquiatria, HCFMUSP, São Paulo, SP, Brazil. ${ }^{25}$ Instituto Nacional de Biomarcadores em Neuropsiquiatria (INBioN), Departamento e Instituto de Psiquiatria, HCFMUSP, São Paulo, SP, Brazil.
\end{abstract}

\begin{abstract}
Electrical and magnetic brain stimulation techniques present distinct mechanisms and efficacy in the acute treatment of depression. This was an umbrella review of meta-analyses of randomized controlled trials of brain stimulation techniques for managing acute major depressive episodes. A systematic review was performed in the PubMed/MEDLINE databases from inception until March 2020. We included the English language meta-analysis with the most randomized controlled trials on the effects of any brain stimulation technique vs. control in adults with an acute depressive episode. Continuous and dichotomous outcomes were assessed. A Measurement Tool to Assess Systematic Reviews-2 was applied and the credibility of evidence was assessed using the Grading of Recommendations Assessment, Development and Evaluation framework. Seven meta-analyses were included (5,615 patients), providing evidence for different modalities of brain stimulation techniques. Three meta-analyses were evaluated as having high methodological quality, three as moderate, and one as low. The highest quality of evidence was found for high frequency-repetitive transcranial magnetic stimulation (rTMS), transcranial direct current stimulation, and bilateral rTMS. There is strong clinical research evidence to guide future clinical use of some techniques. Our results confirm the heterogeneity of the effects across these techniques, indicating that different mechanisms of action lead to different efficacy profiles.
\end{abstract}

Keywords: Brain stimulation; umbrella review; depression; quality of evidence; GRADEpro

Correspondence: Laís Boralli Razza, Serviço Interdisciplinar de Neuromodulação, Rua Dr. Ovidio Pires de Campos, $785,2^{\circ}$ andar, Ala Sul, Instituto de Psiquiatria, CEP 05403-000, São Paulo, SP, Brazil.

E-mail: lais.razza@usp.br

Submitted May 25 2020, accepted Jul 17 2020, Epub Oct 232020.
How to cite this article: Razza LB, Afonso dos Santos L, Borrione L, Bellini H, Branco LC, Cretaz E, et al. Appraising the effectiveness of electrical and magnetic brain stimulation techniques in acute major depressive episodes: an umbrella review of meta-analyses of randomized controlled trials. Braz J Psychiatry. 2021;43:514-524. http://dx.doi.org/10.1590/1516-4446-2020-1169 


\section{Introduction}

Depression is one of the most important global health problems, affecting at least 264 million people worldwide, and ranking third in years lived with disability. ${ }^{1}$ Current psychiatric guidelines for the treatment of depression recommend antidepressants and cognitive-behavioral therapy as first-line interventions. ${ }^{2,3}$ However, pharmacotherapies are associated with modest remission rates and discontinuation due to side effects, ${ }^{4}$ while psychotherapy has a modest effect size and is not readily available for the majority of the world's population. ${ }^{5}$

Brain stimulation techniques are non-pharmacological and non-psychotherapeutic interventions that could fill the gaps in mainstream treatments. ${ }^{6,7}$ These techniques consist of applying electrical current or magnetic fields to modulate neural networks and affect neural plasticity in order to restore or enhance brain function.

Brain stimulation techniques are commonly divided into two main classifications: invasive and non-invasive brain stimulation (NIBS). Invasive techniques such as deep brain stimulation (DBS) and vagus nerve stimulation (VNS) require sedation and surgical procedures. ${ }^{8} \mathrm{How}-$ ever, NIBS techniques use non-implantable devices, do not require sedation or anesthesia, and are applied with intermittent protocols. Hence, the most common form of NIBS is transcranial electric stimulation, whose most widespread variants are transcranial direct current stimulation (tDCS), ${ }^{9}$ and repetitive transcranial magnetic stimulation (rTMS). rTMS is commonly performed as high frequency-rTMS (HF-rTMS) over the left dorsolateral prefrontal cortex (DLPFC) or low frequency-rTMS (LFrTMS) over the right DLPFC. Both procedures can be applied in the same session, and this procedure is described as bilateral rTMS (BL-rTMS). ${ }^{10}$ More recently, other forms of rTMS have been investigated, such as theta-burst stimulation (TBS), ${ }^{11}$ which can be applied intermittently (iTBS) or continuously (cTBS), and both can be applied either unilaterally or bilaterally (BL-TBS); deep TMS (dTMS), which may stimulate deeper (sub)cortical structures; synchronized TMS (sTMS), which uses a lowfield magnetic stimulation synchronized to an individual's alpha frequency ${ }^{12}$; accelerated TMS (aTMS), consisting of multiple sessions in the same day ${ }^{13}$; and priming TMS, employed to "prime" the brain with excitatory (i.e., HFrTMS) stimulation over the right DLPFC to enhance the effects of inhibitory (i.e., LF-rTMS) stimulation over the same region, according to the principle of homeostatic neuroplasticity. Finally, convulsive interventions are neurostimulation techniques that require the use of anesthesia/sedation during their procedure; these include electroconvulsive therapy (ECT) and magnetic seizure therapy (Table 1).

The clinical rationale for neurostimulation techniques in major depressive disorder is that they modulate neuronal circuits and networks, which can undergo potentially reversible alterations in function. For more than two decades, the DLPFC has been the main cortical target for NIBS in depression. ${ }^{14-17}$ This brain area is a key hub of the frontoparietal network, which has been implicated in the regulation of a multitude of processes, such as decision-making, working memory, and attention, and has been found to be hypoactive in depression. ${ }^{18}$ Hypoactivity of the frontoparietal network is associated with hyperactivity of the default mode network, which may promote depressive behaviors and cognitions such as negative bias, self-referential processing, and rumination. ${ }^{19}$ By stimulating the DLPFC, neuromodulation techniques can increase frontoparietal network activity and/or a concomitant down-regulation of default mode network activity, which could contribute to improvement in depressive symptoms. ${ }^{20}$

Meanwhile, invasive techniques like DBS can modulate cortical regions, mainly by inhibiting hyperactivity in limbic-cortical connections, which are implicated in the etiology of major depression, ${ }^{21}$ while VNS stimulates vagal afferent fibers in the neck, modulating the neurocircuits involved in depression through a "bottom-up" approach. ${ }^{22}$ Convulsive therapies are based on the principle of inducing a generalized seizure with therapeutic properties. ${ }^{23}$

Brain stimulation techniques not only have specific neurophysiological actions, but present distinct efficacy profiles for the acute treatment of depression. ${ }^{24}$ Therefore, the aim of this study was to summarize evidence on the therapeutic efficacy of brain stimulation techniques to

Table 1 Summary of the brain stimulation techniques

\begin{tabular}{lll}
\hline Intervention & Principle of action & Sedation \\
\hline tDCS & Low electric current (1-2 mA) applied through two rubber electrodes placed on the scalp. & Not necessary \\
& The current passes through the skin, tissue, and skull to reach the brain and is capable of \\
modulating cortical excitability and brain networks.
\end{tabular}

rTMS

Technique that delivers electrical stimuli through the scalp using a coil. When in contact with conductive tissues (such as the brain), the magnetic field that passes through the coil induces

Not necessary a secondary electric field that can generate action potentials and induce plasticity.

DBS

An electrode is stereotactically implanted in a pre-established target in the brain. The electrode is
connected to a pacemaker-like device, which is implanted subcutaneously on the chest wall.

Necessary When turned on, the electrode delivers high frequency electrical stimulation. a seizure with therapeutic properties.

Necessary

DBS = deep brain stimulation; ECT = electroconvulsive therapy; rTMS = repetitive transcranial magnetic stimulation; tDCS = transcranial direct current stimulation. 
treat depression through an umbrella review. An umbrella review systematically evaluates the information from multiple meta-analyses of a particular topic and can provide a high-level overview. Although previous guidelines ${ }^{25,26}$ have already addressed evidence on the efficacy of multiple brain stimulation techniques, none have used the Grading of Recommendations Assessment, Development and Evaluation (GRADE) framework ${ }^{27}$ and an umbrella review method. GRADE is a well-structured online program that offers organized and systematic steps for developing and presenting evidence and recommendations.

\section{Methods}

In June 2019, the Brazilian Association of Psychiatry and the Brazilian Journal of Psychiatry convened a group of national specialists in the field of neuromodulation to produce evidence-based guidelines on brain stimulation techniques for treating depression.

\section{Search strategy}

We performed a systematic review based on an electronic database search according to PRISMA ${ }^{28}$ and Cochrane Group $^{29}$ guidelines. The study was registered in PROSPERO, the international prospective register of systematic reviews (reference CRD 42019143090).

Two independent authors (LBR and LAS) searched for systematic reviews and meta-analyses in the PubMed/ MEDLINE databases from inception until March 2020 (the specific search terms can be found in Appendix 1, available as online-only supplementary material). The same investigators screened the titles/abstracts of the retrieved references for eligibility and examined the fulltext of potentially eligible studies. Any disagreements were discussed with the co-authors and resolved through consensus.

\section{Eligibility criteria}

We included meta-analyses of pairwise, network, or individual patient data with the largest number of randomized controlled trials (RCTs) for each technique. Only meta-analyses in English that evaluated the effects of any brain stimulation technique vs. sham for the treatment of depression were considered. We included studies enrolling adult patients ( $\geqslant 18$ years old) with an acute depressive episode that was associated with a diagnosis of major depressive disorder. Meta-analyses evaluating bipolar disorder were also included when this sample was less than $10 \%$ of the overall sample and it otherwise consisted of patients diagnosed with unipolar depression.

We included meta-analyses evaluating the effects of any invasive, NIBS, or convulsive therapies and sham techniques of each intervention for the treatment of depression. The NIBS techniques were: transcranial electric stimulation (tDCS, transcranial pulsed current stimulation [tPCS], transcranial random noise stimulation [tRNS], auricular/transcutaneous VNS [atVNS/tVNS] and transcranial alternating current stimulation [tACS] and transcranial magnetic stimulation [TMS - BL-rTMS, LFrTMS, HF-rTMS, BL-TBS, cTBS, iTBS, dTMS, aTMS, sTMS, and priming TMS]). The convulsive therapies were magnetic seizure therapy and ECT. Finally, the invasive brain stimulation techniques were DBS and VNS. Only direct comparisons between the active and sham techniques were included.

We included only the meta-analyses (pairwise, network, or individual patient data) that evaluated two or more RCTs per technique, and only randomized, doubleblind trials or those that presented the results in a separate analysis and reported categorical (response and/or remission rates) and/or continuous (improvement in pre- and post-test depression scores) outcomes.

The exclusion criteria were other types of study designs, studies with duplicate data, and those written in languages other than English. We also excluded the less recent meta-analyses when two or more metaanalyses assessed the same number of RCTs.

\section{Data extraction}

The extracted data were input into the GRADE system. ${ }^{27}$ GRADE is available online at www.gradepro.org. The extracted variables were: 1) number of participants (active and control groups); 2) number of responders; 3) number of remitters; 4 ) relative (odds ratio [OR], risk ratio, or hazard ratio) or absolute effects.

Four authors were involved in the data extraction process (LBR, LAS, LB, and ARB) and two (LBR and LAS) checked the extracted data independently. Data that could not be determined from the original publications were requested from the corresponding authors via e-mail.

\section{Quality assessment}

We assessed the quality of the studies using A Measurement Tool to Assess Systematic Reviews (AMSTAR)-2. Validated for this purpose ${ }^{30}$ AMSTAR-2 uses a checklist of 16 domains to evaluate systematic reviews of RCTs. The checklist can rate the overall quality of metaanalyses, categorizing them as high (no weaknesses or one non-critical weakness), moderate (more than one non-critical weakness), low (one critical flaw with or without non-critical weaknesses), and critically low (more than one critical flaw with or without non-critical weaknesses). AMSTAR-2 is available online at www. amstar.ca.

\section{Data analysis}

We adopted the GRADE evidence profile and Summary of Findings tables ${ }^{31}$ to evaluate the overall quality of evidence of each brain stimulation technique. We separately rated the quality of evidence for dichotomous (response and remission) and/or continuous (improvement in depression scales) outcomes for any direct comparison between interventions across the included studies. We created an independent Summary of Findings table per technique. 
The studies' certainty assessment (study design, risk of bias, inconsistency, indirectness of evidence, and imprecision) rated the risk of individual features as either not serious, serious, or very serious, and provided Summary of Findings table information (number of patients for intervention and control groups, and the relative and absolute effects). Based on Summary of Findings table information, the level of evidence by outcome was automatically determined through certainty assessment, being graded as high (further research is very unlikely to change confidence in the estimate of effect), moderate (further research is likely to have an important impact on confidence in the estimate of effect and may change the estimate), low (further research is very likely to have an important impact on confidence in the estimate of effect and is likely to change the estimate), or very low (any estimate of effect is very uncertain). ${ }^{32}$

Moreover, per GRADE's recommendation, we evaluated the magnitude of an effect as none, large, or very large. Large and very large effects are respectively defined as a relative risk $>2$ or $<0.5$ and relative risk $>5$ or $<0.2$ if no plausible confounder was identified. The level of evidence can be increased if the effects are large or very large. Here, we considered that not only should the effect size have been large, but that the confidence interval should have been narrow to increase the level of evidence.

\section{Results}

Out of 2,565 references identified, 2,558 were excluded for diverse reasons (Figure 1). A total of seven meta-analyses (two network and five pairwise meta-analyses) ${ }^{10,24,33-37}$ met our inclusion criteria, providing information on 12 direct comparisons between active brain stimulation techniques and their respective sham counterparts in a total of 5,615 patients (Table 2 ). The quality of evidence of active vs. sham interventions was evaluated through

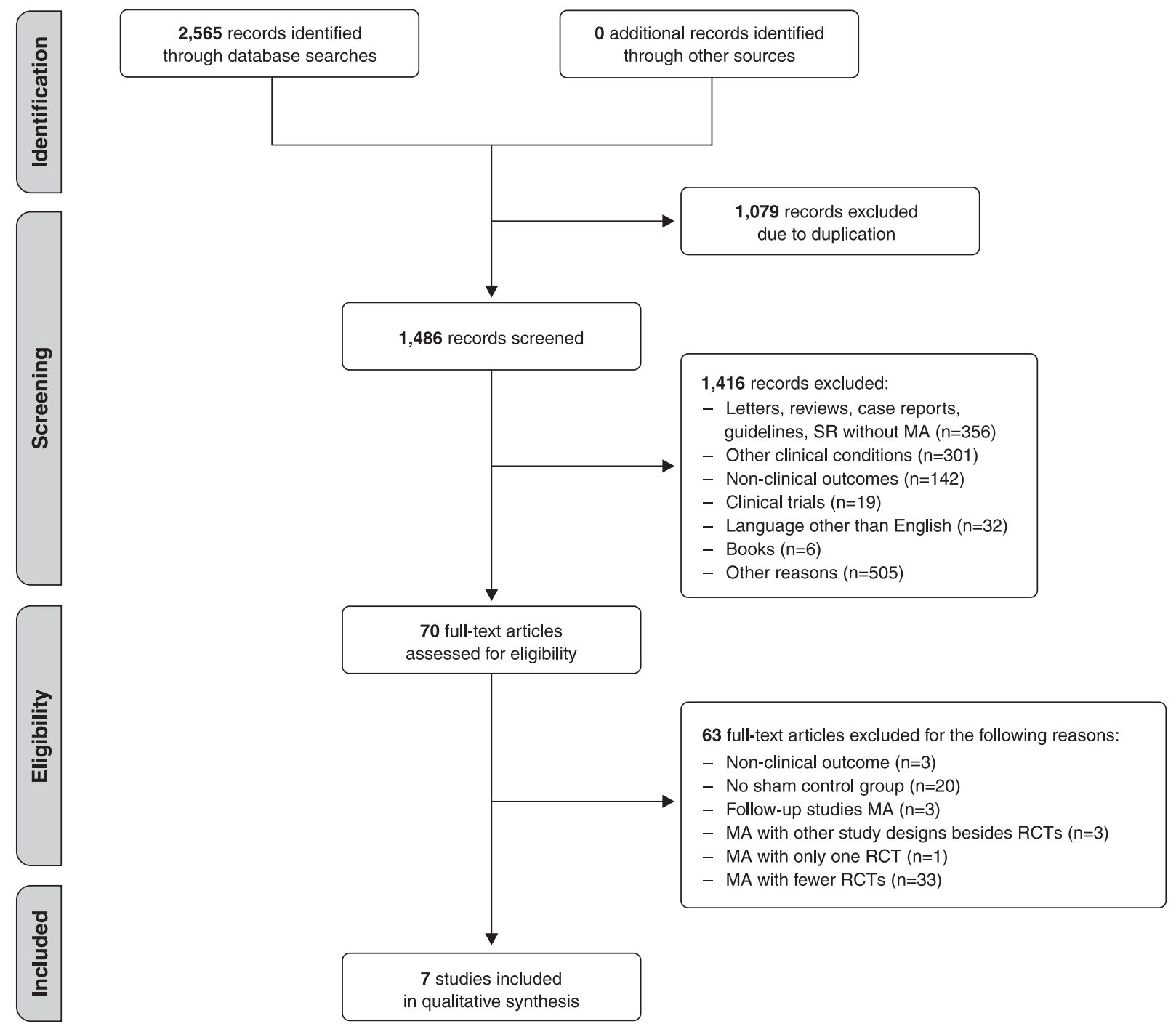

Figure 1 Flowchart of the included meta-analyses. MA = meta-analysis; RCTs = randomized controlled trials; SR = systematicreview. 
the GRADEpro system (see Appendix 2, available as online-only supplementary material, for further information). The AMSTAR-2 scores for high-, moderate-, and low-quality meta-analyses were $42.9,42.9$, and $14.2 \%$, respectively. No meta-analysis was considered to have critically low methodological quality (Appendix 3, available as online-only supplementary material).

Since our search failed to retrieve eligible meta-analyses on tPCS, tRNS, atVNS, tVNS, tACS, cTBS, magnetic seizure therapy, and VNS for depression treatment, no conclusions have been drawn.

\section{Evidence grading: transcranial electric stimulation}

\section{tDCS}

The included meta-analysis ${ }^{37}$ explored continuous (depression improvement) and dichotomous (response and remission) effects, showing that active tDCS was superior to the comparison groups across examined outcomes per random-effect $(\mathrm{k}=25, \mathrm{~g}=0.46,95 \%$ confidence interval $[95 \% \mathrm{Cl}]$ 0.22-0.7) and OR calculation for response and remission $(\mathrm{k}=18, \mathrm{OR}=2.28,95 \% \mathrm{Cl} 1.52-3.42$, and $\mathrm{k}=18, \mathrm{OR}=2.12,95 \% \mathrm{Cl} 1.42-3.16$, respectively). According to AMSTAR-2, the overall methodological quality of this meta-analysis was high. Based on these results, and since the meta-analysis presented no serious risks in the certainty assessment, the intervention was graded as having high certainty of evidence for the three evaluated outcomes (Table 3).

\section{Evidence grading: TMS}

HF-rTMS to the left DLPFC vs. sham

The included meta-analysis ${ }^{24}$ on HF-rTMS to the left DLPFC assessed 40, 31, and 37 RCTs investigating response, remission, and continuous outcomes, respectively. According to AMSTAR-2, the overall methodological quality of the study was moderate. The pooled rate ratios for response and remission were $3.17(95 \% \mathrm{Cl} 2.29$ $4.37)$ and $2.67(95 \% \mathrm{Cl} 1.79-4.00)$, respectively, favoring active HF-rTMS. The standardized mean difference (SMD) was $0.54(95 \% \mathrm{Cl} 0.33-0.76)$, which also favored the real intervention. The technique was graded as high certainty of evidence compared to sham for the three outcomes (Table 3), mainly due to the large effect favoring active intervention.

\section{LF-rTMS to the right DLPFC vs. sham}

The meta-analysis evaluating LF-rTMS to the right DLPFC included eight RCTs in the analyses. ${ }^{33}$ All eight RCTs assessed response and six evaluated remission, showing low overall methodological quality according to the AMSTAR-2. The intervention group had a $3.35(95 \%$ $\mathrm{Cl}$ 1.34-8.02) times greater odds of response than the sham group, as well as a $4.76(95 \% \mathrm{Cl} 2.13-10.64)$ times greater odds of remission than sham. Although two outcomes showed high effects, we did not grade response and remission rates as "very large" due to the 
Table 3 Quality of evidence for response, remission, and continuous outcomes

\begin{tabular}{lccc}
\hline Interventions & Response & Remission & Continuous \\
\hline tDCS & High & High & High \\
HF-rTMS (IDLPFC) & High & High & High \\
BL-rTMS & High & High & - \\
LF-rTMS (rDLPFC) & Moderate & Moderate & Moderate \\
aTMS & Low & - & Moderate \\
ECT & - & - & Low \\
dTMS & Low & Low & Low \\
iTBS & Low & Low & Low \\
LF-rTMS (IDLPFC) & Low & Low & Low \\
STMS & Low & Low & Low \\
DBS & Low & - & -
\end{tabular}

aTMS = accelerated transcranial magnetic stimulation; BL-rTMS = bilateral-repetitive TMS; BL-TBS = bilateral theta-burst stimulation; DBS = deep brain stimulation; dTMS = deep TMS; ECT = electroconvulsive therapy; HF-rTMS = high frequency-repetitive TMS; iTBS = intermittent TBS; IDLPFC = left dorsolateral prefrontal cortex; LF-rTMS = low frequency-rTMS; rDLPFC = right DLPFC; sTBS = synchronized TBS; tDCS = transcranial direct current stimulation.

width of the $\mathrm{Cl}$. The study was graded as moderate quality of evidence for response and remission (Table 3).

\section{BL-rTMS vs. sham}

The meta-analysis evaluating BL-rTMS vs. sham included 11 RCTs. ${ }^{10}$ All 11 RCTs reported response rates and eight reported remission rates. The AMSTAR-2 score indicated high overall methodological quality.

The pooled rate ratios for remission and response rates were $4.22(95 \% \mathrm{Cl} 1.96-9.05)$ and $3.96(95 \% \mathrm{Cl} 2.37-$ $6.60)$, favoring BL-rTMS for both outcomes. The certainty of evidence for the remission outcome was downgraded due to the large $\mathrm{Cl}$ width. Therefore, the remission rate was graded as moderate quality, while the response rate was graded as high quality (Table 3 ).

Intermittent TBS vs. sham

The meta-analysis included in our study evaluated three RCTs using iTBS. ${ }^{24}$ The three RCTs evaluated response, while two evaluated remission and continuous outcome. The meta-analysis showed moderate overall methodological quality.

The iTBS protocol showed significant antidepressant efficacy for response $(\mathrm{k}=3, \mathrm{OR}=3.20,95 \% \mathrm{Cl} 1.45-7.08)$ and remission $(\mathrm{k}=02, \mathrm{OR}=3.30,95 \% \mathrm{Cl} 1.38-7.90)$. The effect of the continuous outcome was SMD $=0.41(95 \% \mathrm{Cl}$ $0.35-1.17)$. The intervention was graded as low certainty of evidence (Table 3 ) due to the small sample size and the large $\mathrm{Cl}$ width for response and remission rates, as well as the small magnitude for the continuous outcome.

\section{BL-TBS vs. sham}

The meta-analysis comparing bilateral TBS vs. sham stimulation evaluated response, remission, and continuous outcome in its analyses; however, only the response rate was reported in more than one RCT. ${ }^{24}$ The AMSTAR-2 score indicated moderate overall methodological quality.

The response rate was $\mathrm{k}=2$, OR $=4.44,95 \% \mathrm{Cl} 1.47-$ 13.41, showing superior efficacy than to the sham intervention (Table 3 ). Although the effect of the active intervention was superior to sham, the quality of evidence was graded as low due to the small sample size and the large $\mathrm{Cl}$ width.

\section{dTMS vs. sham}

The selected meta-analysis included two RCTs that, according to the AMSTAR-2 results, presented moderate overall methodological quality. Both RCTs assessed continuous and dichotomous outcomes. ${ }^{24}$ Compared to the sham intervention, dTMS resulted in no significant improvements in response or remission rates $(\mathrm{k}=2, \mathrm{OR}=$ $1.87,95 \% \mathrm{Cl} 0.78-4.49$ and $\mathrm{k}=2, \mathrm{OR}=2.21,95 \% \mathrm{Cl} 0.95-$ 5.18 , respectively). The continuous outcome was $\mathrm{k}=2$, SMD $=-0.36,95 \% \mathrm{Cl}-1.22-0.50$, showing no efficacy for the active intervention. Although the effect of dTMS on depression was significant for the continuous outcome, the quality of evidence for the three outcomes was graded as low due to the small sample sizes (Table 3).

\section{sTMS vs. sham}

The included meta-analysis evaluated results from two RCTs. ${ }^{24}$ The meta-analysis had moderate methodological quality according to AMSTAR-2. None of the three outcomes (response, remission, or continuous effects) presented significant results compared to the sham intervention $(\mathrm{k}=2, \mathrm{OR}=2.09,95 \% \mathrm{Cl} 0.76-5.77, \mathrm{k}=2$, $\mathrm{OR}=1.59,95 \% \mathrm{Cl} 0.52-4.81$, and $\mathrm{k}=2, \mathrm{SMD}=-0.57$, $95 \% \mathrm{Cl}-1.43-0.29$, respectively).

The intervention was graded as having low quality of evidence for each of the three outcomes (Table 3 ).

aTMS vs. sham

The meta-analysis evaluating the effects of aTMS for depression treatment included three RCTs. Only response and continuous effects were evaluated. ${ }^{36}$ AMSTAR-2 revealed an overall high methodological quality. The response rate to active aTMS was not significantly different than sham aTMS. The OR of response was 3.13 compared to sham $(\mathrm{k}=3,95 \% \mathrm{Cl} 0.98-9.97)$. A small effect size was found for continuous outcome $(\mathrm{k}=3, \mathrm{~g}=0.39,95 \% \mathrm{Cl}$ $0.005-0.28$ ). Due to the small sample size and the large $\mathrm{Cl}$ 
width for response rate, the quality of evidence for this outcome was graded as low, while the continuous outcome was considered as having moderate certainty of evidence (Table 3).

\section{LF-rTMS to the left DLPFC vs. sham}

Few RCTs have also evaluated the effects of LF-rTMS applied to the left DLPFC. Four studies were evaluated in the included meta-analysis, ${ }^{24}$ which presented moderate overall methodological quality according to AMSTAR-2 scores. All four RCTs reported remission and response outcomes, and only three reported a continuous outcome.

LF-rTMS to the left DLPFC was not associated with significant improvement in response and remission rates $(\mathrm{k}=4, \mathrm{OR}=1.10,95 \% \mathrm{Cl} 0.21-5.87$, and $\mathrm{k}=4, \mathrm{OR}=1.02$, $95 \% \mathrm{Cl}$ 0.17-6.02, respectively). Furthermore, no evidence of efficacy was found for the continuous outcome $(\mathrm{k}=2, \mathrm{SMD}=0.03,95 \% \mathrm{Cl}-0.76-0.81)$.

This protocol was evaluated as having low certainty of evidence for all evaluated outcomes (Table 3).

\section{Evidence grading: convulsive therapies}

ECT vs. sham

The meta-analysis evaluating active ECT vs. sham stimulation included six RCTs with only continuous outcomes. ${ }^{35}$ The study had moderate overall methodological quality according to the AMSTAR-2 score.

The continuous outcome for active ECT was significantly better than sham $(k=6$, standardized effect size $=$ $-0.98,95 \% \mathrm{Cl}-1.27--0.54)$. Heterogeneity was not reported. Although the effect was high for depression treatment, the evidence quality for this technique was moderate due to the small sample sizes of the included trials (Table 3 ).

\section{Evidence grading: invasive brain stimulation}

\section{DBS}

The selected meta-analysis included RCTs and nonRCTs in its analysis. We could only access the results of a sub-analysis evaluating two RCTs investigating the effects of DBS implanted in the internal/ventral capsules. The meta-analysis had moderate methodological quality ${ }^{34}$ and evaluated continuous and response outcomes. ${ }^{34}$ The active treatment group had a significantly higher response than the sham group $(\mathrm{k}=2, \mathrm{OR}=4.85,95 \% \mathrm{Cl}$ $0.52-45.32$ ), but no significant reduction in mean depression score $(\mathrm{k}=2, \mathrm{SMD}=-0.46,95 \% \mathrm{Cl}-1.78-0.87)$.

Despite the positive results for DBS vs. sham, the small sample size prevented the intervention from having maximum quality of evidence. The two outcomes were thus graded as having low certainty of evidence (Table 3).

\section{Discussion}

Our systematic review on meta-analyses of RCTs investigating invasive, non-invasive, and convulsive neuromodulation techniques for acute treatment of major depressive episode in adults found seven eligible studies (two network and five pairwise meta-analyses), including a total of 5,615 patients diagnosed with major depressive episode who were allocated in a total of 12 interventions and their respective sham groups. The active interventions were tDCS, HF-rTMS to the left DLPFC, LF- to the right DLPFC, BL-rTMS, iTBS, BL-TBS, dTMS, sTMS, aTMS, LF-rTMS to the left, ECT, and DBS.

To the best of our knowledge, this current umbrella review is the first effort to review the level of evidence across a large spectrum of biophysical neuromodulation techniques for the treatment of depression according to the GRADE system. This system allowed us to identify the quality of evidence of each technique in a standardized fashion. Thus, we could evaluate the certainty of evidence for response, remission and/or continuous outcomes for different brain stimulation interventions.

Across the included meta-analyses, HF-rTMS to the left DLPFC and tDCS had the highest quality of evidence in terms of response, remission, and continuous antidepressant outcomes compared to sham. Both treatment types also had large effect sizes for response and remission and a moderate effect size for depression ratings. In fact, both techniques have been extensively investigated as antidepressant interventions in the past two decades, ${ }^{15,38,39}$ and both were assessed in over one thousand patients. ${ }^{37,40}$ rTMS techniques for major depressive disorder have been assessed in more than 50 RCTs. The vast majority applied HF-rTMS to the left DLPFC, finding positive results for the active intervention. ${ }^{10,24,41}$ tDCS has been evaluated for depression in more than 20 sham-controlled trials since 2000 . Two large RCTs evaluating its therapeutic effect for major depressive disorder found superior antidepressant efficacy compared to sham. ${ }^{17,42}$ Moreover, an effect favoring the active intervention was also found in a study with bipolar depressive patients. ${ }^{43}$ Although there was high heterogeneity across tDCS studies, ${ }^{44}$ the included meta-analysis showed very consistent effects overall for recent findings. Thus, the large number of RCTs and patients assessed in our analyses reinforce our confidence that high quality data provide evidence for the antidepressant efficacy of rTMS and tDCS compared to sham.

So far, no RCT has directly compared the clinical efficacy of HF-rTMS and tDCS for depression. However, the network meta-analyses included in this study showed that both techniques present similar effects for response and continuous outcomes. ${ }^{24}$ Even though both techniques had high quality of evidence, several studies have concluded that tDCS should only be used to treat moderate depression since, unlike HF-rTMS, it is not efficacious against treatment-resistant depression. ${ }^{45-47}$ However, tDCS is performed with a portable device and has been investigated for self-application. ${ }^{48,49}$ The portability and feasibility of tDCS can increase treatment adherence and allow concomitant use with other interventions (such as cognitive behavioral therapy or cognitive training therapy), possibly improving the effects of tDCS as a monotherapy. ${ }^{50}$

Our results showed that BL-rTMS also had a high level of efficacy for response and remission, again with large 
effect sizes. Unfortunately, continuous outcomes were not assessed by the included network meta-analysis. Individual studies have already shown that the technique is more efficacious than sham intervention, for instance. ${ }^{51}$ The difference between BL-rTMS and HF-rTMS is that the bilateral intervention involves the simultaneous or subsequent application of HF and LF protocols over the contralateral brain regions in the same session. ${ }^{9}$ Thus, considering the quality of evidence, one advantage of applying HF-rTMS instead of BL-rTMS in clinical practice is its shorter duration (20 minutes vs. 50 minutes, on average).

Of the three most common rTMS protocols in depression (BL, HF, and LF), LF-rTMS over the right DLPFC presented the lowest quality of evidence, with moderate certainty of evidence for the assessed outcomes (response and remission rates). Although our findings showed large effect sizes for LF-rTMS vs. sham for response and remission, the sample was small, which was the main reason for downgrading confidence about the efficacy of this technique. However, in clinical practice, LF-rTMS is advantageous due to its shorter protocol than BL-rTMS. LF-rTMS sessions are very similar duration to the HF-rTMS protocol, considering standard parameters of $1 \mathrm{~Hz}$ pulses (60 pulses per minute) and 600 to 1,800 LF-rTMS pulses at $10 \mathrm{~Hz}$ delivered in 5 seconds (a "train"), with a 20- to 40 -second interval between trains, and a total of 1,200 to 3,000 HF-rTMS pulses. ${ }^{9}$ Thus, the protocol durations for the interventions are approximately 20 and 40 minutes, respectively. In terms of clinical presentation of depression symptoms, the literature shows ${ }^{52}$ that LF-rTMS can be more effective for patients with a higher degree of comorbid anxiety/panic symptoms, while HF-rTMS appears to be more suitable for patients with a lack of motivation and cognitive impairment.

Moderate quality of evidence was also found for continuous outcomes of aTMS and ECT. These findings suggest that the techniques can probably improve depressive symptoms, but their efficacy for achieving response and/or remission is unclear. Despite a large effect size for response and a small effect size for continuous outcome, aTMS was considered to have a low level of evidence for response mainly due to the small sample size in the included study. ${ }^{36}$ Actually, a meta-analysis investigating the effects of aTMS for depression showed superior response and remission rates for active stimulation compared to sham. ${ }^{24}$ However, the findings of the studies included in this meta-analysis were heterogeneous. This suggests that, in fact, there is still limited evidence about the effects of aTMS on dichotomous outcomes.

Our findings about ECT should be interpreted with caution due to the natural bias in ECT research, i.e., only a few sham-controlled RCTs have evaluated the effects of ECT for depression. ${ }^{53-55}$ Thus, our meta-analysis could only evaluate six RCTs from the last few decades that did not include data on dichotomous outcomes (response and remission). ${ }^{35}$ The ECT evidence was of moderate quality for continuous outcomes. Although our results reflect the findings of well-controlled trials, it is important to emphasize that ECT is an established technique for depression treatment, ${ }^{56,57}$ and most RCTs on ECT have included an active comparator (mainly antidepressant drugs) instead of a sham protocol, and were thus not included in the present review. In current research, ECT has usually been compared to active controls, since its efficacy for depression has already been proven superior to sham in prior RCTs. Moreover, the trend of comparing ECT to other active interventions is due to the ethical concerns of sham ECT treatment, since the required anesthesia also incurs a risk for patients. ${ }^{58}$

It must be pointed out that ECT is more efficacious than even active interventions in reducing depressive symptoms. ${ }^{59,60}$ For instance, according to a recent network meta-analysis on the efficacy of non-surgical brain stimulation (rTMS and tDCS) for the acute treatment of depression, bitemporal and high-dose right unilateral ECT had the highest probability of efficacy in terms of a response. $^{24}$

Despite having large effects for response and medium effects for depression ratings, DBS had a low quality of evidence for these outcomes, being based on only two RCTs, and no data were available to assess remission rates for this intervention. DBS is an invasive neuromodulation technique that was first implemented in neuropsychiatric practice to treat severe, treatment-resistant obsessive-compulsive disorder and has shown promising results. ${ }^{61,62}$ More recently, there has been significant interest in using DBS for treatment-resistant depression. ${ }^{63,64}$ In general, DBS has been found efficacious for treating non-responders to several other treatments ${ }^{65,66}$ and had a low risk of relapse. ${ }^{67}$ However, few patients have been assessed, probably because DBS requires surgery and is more complex than NIBS or convulsive therapies, ${ }^{25}$ which downgraded its quality of evidence for depression treatment. DBS might be an efficient intervention for treatment-resistant depression patients, but its widespread use is limited by its high cost and invasiveness.

Our analyses also showed that two techniques approved by the US Food and Drug Administration (FDA), dTMS (an almost large effect size for response and a large effect size for remission, but a small effect size for continuous outcome) and iTBS (a large effect size for response and remission, but a small effect size for continuous outcome), still had low certainty of evidence regarding their use in antidepressant treatment according to the GRADE system. iTBS received FDA clearance for treatment-resistant depression after a recent trial showed it to be non-inferior to HF-rTMS for reducing depressive symptoms. ${ }^{16}$ dTMS was also approved for treatment-resistant depression after a $\mathrm{RCT}^{68}$ demonstrated that dTMS was significantly better than sham treatment for continuous, response, and remission outcomes. Although dTMS and iTBS are FDA-approved and promising in terms of antidepressant efficacy, ${ }^{69-71}$ our analyses showed that there are still very few shamcontrolled trials for these interventions, which prevents any statement about their efficacy for depression. The same is true for BL-iTBS. However, special attention should be given to TBS protocols, given their short protocol, which can range from 2 to 10 minutes per session. ${ }^{72}$ Finally, according to our findings, LF-rTMS to the left DLPFC and 
sTMS have been investigated less than standard HF rTMS protocols and have low quality of evidence for all assessed outcomes.

Although safety was not systematically assessed in this review, the literature shows that NIBS techniques, such as tDCS and rTMS, are deemed safe for the treatment of depression, ${ }^{25,73,74}$ presenting few and tolerable adverse effects. To date, no serious adverse effects have been reported for tDCS treatment, ${ }^{75,76}$ and less than $0.01 \%$ of subjects have a seizure episode during rTMS prefrontal stimulation. ${ }^{77}$ ECT also presents a favorable safety profile when the guidelines are followed. ${ }^{25}$ In fact, its major risks are associated with anesthesia, ${ }^{78}$ which can lead to serious adverse effects in patients with clinical comorbidities. In addition, long-term short-term memory impairment has been reported in some patients who underwent ECT ${ }^{79}$ Finally, there are still no evidence-based safety recommendations for DBS, ${ }^{62}$ although the procedure involves all the risks inherent to a neurosurgical procedure. ${ }^{80}$

Our review has several limitations that should be considered. First, our search was performed only in the PubMed database and we only included meta-analyses in English. This decision was made a priori, since the overwhelming majority of meta-analyses are published in English and are properly indexed in PubMed. In addition, when more than one meta-analysis was found regarding a specific intervention, we included only the most recent one. Second, since the included meta-analyses evaluated only acute effects, we could not assess the level of evidence for the prolonged effects (maintenance phase) of each intervention in the treatment of depression. Moreover, we restricted our analysis to active interventions compared to sham protocols, but not to other active comparators. This can be particularly relevant regarding ECT, since very few studies have investigated its effectiveness compared to sham control conditions. In addition, this review did not evaluate techniques specifically developed for treatment-resistant depression patients, for whom ECT is recommended as a first-line treatment. Third, the meta-analyses may have included RCTs with different active parameters for each technique (e.g., number of pulses, number of sessions, etc.). Fourth, we did not cover the safety and tolerability of each intervention, which is a major limitation. Finally, we believe that our conclusions are limited by a lack of comparative studies in this field, e.g., no recent RCTs have compared NIBS with ECT or even invasive brain stimulation.

In conclusion, the results of this umbrella review, which included seven meta-analyses evaluating the effects of 12 brain stimulation techniques for depression treatment in 5,615 patients, revealed that a large body of data supports HF-rTMS to the left DLPFC, BL-rTMS, and tDCS at the highest level of evidence, based on large sample sizes and with very consistent effects across the most recent RCTs. The certainty of evidence of most interventions was downgraded as a result of small sample sizes. The quality of evidence for ECT, an established technique for depression, was considered moderate, while two FDA-approved techniques (iTBS and dTMS) had low quality of evidence. Therefore, our findings confirm that brain stimulation techniques have matured, becoming important treatment alternatives in the treatment of depressive disorders, and could soon join pharmacological treatments and psychotherapy as standard intervention options.

\section{Acknowledgements}

LBR is supported by the Fundação de Amparo à Pesquisa do Estado de São Paulo (FAPESP; grant no. 2019/ 07256-7). CUC has received grant support from the Berlin Institute of Health, the National Institute of Mental Health, the Patient Centered Outcomes Research Institute, and the Thrasher Foundation. ARB receives grants from the Conselho Nacional de Desenvolvimento Científico e Tecnológico (CNPq PQ-1B) and from Programa de Incentivo à Produtividade Acadêmica of the Faculdade de Medicina, Universidade de São Paulo (USP).

\section{Disclosure}

CUC has been a consultant and/or advisor to or has received honoraria from Acadia, Alkermes, Allergan, Angelini, Axsome, Gedeon Richter, Gerson Lehrman Group, IntraCellular Therapies, Janssen/J\&J, LB Pharma, Lundbeck, MedAvante-ProPhase, Medscape, Neurocrine, Noven, Otsuka, Pfizer, Recordati, Rovi, Sumitomo Dainippon, Sunovion, Supernus, Takeda, and Teva; has provided expert testimony for Janssen and Otsuka; has served on a Data Safety Monitoring Board for Lundbeck, Rovi, Supernus, and Teva; has received grant support from Janssen and Takeda; has received royalties from UpToDate; and is a stock option holder of LB Pharma. FP is a member of the European Scientific Advisory Board of Brainsway Inc., Jerusalem, Israel; has received speaker's honoraria from Mag \& More $\mathrm{GmbH}$ and the neuroCare Group; his lab has received support with equipment from neuroConn $\mathrm{GmbH}$, Ilmenau, Germany, Mag \& More $\mathrm{GmbH}$, and Brainsway Inc., Jerusalem, Israel. ARB is chief medical advisor of $\mathrm{Flow}^{\mathrm{TM}}$ and has small equity in the company. The other authors report no conflicts of interest.

\section{References}

1 GBD 2016 Disease and Injury Incidence and Prevalence Collaborators. Global, regional, and national incidence, prevalence, and years lived with disability for 328 diseases and injuries for 195 countries, 1990--2016: a systematic analysis for the Global Burden of Disease Study 2016. Lancet. 2017;390:1211-59.

2 Kennedy SH, Lam RW, Mclntyre RS, Tourjman SV, Bhat V, Blier P et al. Canadian Network for Mood and Anxiety Treatments (CANMAT) 2016 clinical guidelines for the management of adults with major depressive disorder: section 3. Pharmacological treatments. Can J Psychiatry. 2016;61:540-60.

3 Parikh SV, Quilty LC, Ravitz P, Rosenbluth M, Pavlova B, Grigoriadis $S$, et al. Canadian Network for Mood and Anxiety Treatments (CANMAT) 2016 clinical guidelines for the management of adults with major depressive disorder: section 2. Psychological treatments. Can J Psychiatry. 2016;61:524-39.

4 Rush AJ, Trivedi MH, Wisniewski SR, Stewart JW, Nierenberg AA, Thase ME, et al. Bupropion-SR, sertraline, or venlafaxine-XR after failure of SSRIs for depression. N Engl J Med. 2006;354: 1231-42. 
5 Keller MB, McCullough JP, Klein DN, Arnow B, Dunner DL, Gelenberg AJ, et al. A comparison of nefazodone, the cognitive behavioralanalysis system of psychotherapy, and their combination for the treatment of chronic depression. N Engl J Med. 2000;342:1462-70.

6 Chris B, Brem AK, Arns M, Brunoni AR, Filipcic I, Ganho-Ávila A, et al. Repetitive transcranial magnetic stimulation treatment for depressive disorders: current knowledge and future directions. Curr Opin Psychiatry. 2019;32:409-15.

7 Brunoni AR, Teng CT, Correa C, Imamura M, Brasil-Neto JP, Boechat RB, et al. Neuromodulation approaches for the treatment of major depression: challenges and recommendations from a working group meeting. Arq Neuropsiquiatr. 2010;68:433-51

8 Klooster DC, de Louw AJ, Aldenkamp AP, Besseling RM, Mestrom $\mathrm{RM}$, Carrette $\mathrm{S}$, et al. Technical aspects of neurostimulation: focus on equipment, electric field modeling, and stimulation protocols. Neurosci Biobehav Rev. 2016;65:113-41.

9 Brunoni AR, Sampaio-Junior B, Moffa AH, Aparicio LV, Gordon P, Klein I, et al. Noninvasive brain stimulation in psychiatric disorders: a primer. Braz J Psychiatry. 2019;41:70-81.

10 Brunoni AR, Chaimani A, Moffa AH, Razza LB, Gattaz WF, Daskalakis ZJ, et al. Repetitive transcranial magnetic stimulation for the acute treatment of major depressive episodes: a systematic review with network meta-analysis. JAMA Psychiatry. 2017;74:143-52.

11 Huang YZ, Edwards MJ, Rounis E, Bhatia KP, Rothwell JC. Theta burst stimulation of the human motor cortex. Neuron. 2005;45:201-6.

12 Leuchter AF, Cook IA, Feifel D, Goethe JW, Husain M, Carpenter LL, et al. Efficacy and safety of low-field synchronized transcranial magnetic stimulation (sTMS) for treatment of major depression. Brain Stimul. 2015;8:787-94.

13 Fitzgerald PB, Hoy KE, Elliot D, McQueen RN, Wambeek LE, Daskalakis ZJ. Accelerated repetitive transcranial magnetic stimulation in the treatment of depression. Neuropsychopharmacology. 2018;43:1565-72.

14 George MS, Wassermann EM, Williams WA, Callahan A, Ketter TA, Basser $P$, et al. Daily repetitive transcranial magnetic stimulation (rTMS) improves mood in depression. Neuroreport. 1995;6:1853-6.

15 Fregni F, Boggio PS, Nitsche MA, Marcolin MA, Rigonatti SP Pascual-Leone A. Treatment of major depression with transcranial direct current stimulation. Bipolar Disord. 2006;8:203-4.

16 Blumberger DM, Vila-Rodriguez F, Thorpe KE, Feffer K, Noda Y, Giacobbe $P$, et al. Effectiveness of theta burst versus high-frequency repetitive transcranial magnetic stimulation in patients with depression (THREE-D): a randomised non-inferiority trial. Lancet. 2018;391: 1683-92.

17 Brunoni AR, Moffa AH, Sampaio-Junior B, Borrione L, Moreno ML, Fernandes RA, et al. Trial of electrical direct-current therapy versus escitalopram for depression. N Engl J Med. 2017;376:2523-33.

18 Kaiser RH, Andrews-Hanna JR, Wager TD, Pizzagalli DA. Largescale network dysfunction in major depressive disorder: a metaanalysis of resting-state functional connectivity. JAMA Psychiatry. 2015;72:603-11.

19 Williams LM. Precision psychiatry: a neural circuit taxonomy for depression and anxiety. Lancet Psychiatry. 2016;3:472-80.

20 Baeken C, De Raedt R. Neurobiological mechanisms of repetitive transcranial magnetic stimulation on the underlying neurocircuitry in unipolar depression. Dialogues Clin Neurosci. 2011;13:139-45.

21 Schlapfer TE, Bewernick BH. Deep brain stimulation for psychiatric disorders--state of the art. Adv Tech Stand Neurosurg. 2009;34:37-57.

22 O'Reardon JP, Cristancho P, Peshek AD. Vagus nerve stimulation (VNS) and treatment of depression: to the brainstem and beyond. Psychiatry (Edgmont). 2006;3:54-63.

23 Pandurangi AK, Fernicola-Bledowski C, Bledowski J. Brain stimulation therapies for psychiatric disorders: the first decade of the new millennium--a review. Asian J -Psychiatr. 2012;5:3-10.

24 Mutz J, Vipulananthan V, Carter B, Hurlemann R, Fu CH, Young AH. Comparative efficacy and acceptability of non-surgical brain stimulation for the acute treatment of major depressive episodes in adults: systematic review and network meta-analysis. BMJ. 2019;364: 11079.

25 Milev RV, Giacobbe P, Kennedy SH, Blumberger DM, Daskalakis ZJ, Downar J, et al. Canadian Network for Mood and Anxiety Treatments (CANMAT) 2016 clinical guidelines for the management of adults with major depressive disorder: section 4. Neurostimulation treatments. Can J Psychiatry. 2016;61:561-75.
26 Lefaucheur JP, Aleman A, Baeken C, Benninger DH, Brunelin J, Di Lazzaro V, et al. Evidence-based guidelines on the therapeutic use of repetitive transcranial magnetic stimulation (rTMS): an update (2014-2018). Clin Neurophysiol. 2020;131:474-528.

27 GRADEpro GDT. GRADEpro guideline development tool [software]. McMaster University; 2015.

28 Liberati A, Altman DG, Tetzlaff J, Mulrow C, Gøtzsche PC, loannidis JP, et al. The PRISMA statement for reporting systematic reviews and meta-analyses of studies that evaluate healthcare interventions: explanation and elaboration. BMJ. 2009;339:b2700.

29 Furlan AD, Pennick V, Bombardier C, van Tulder M; Editorial Board, Cochrane Back Review Group. 2009 updated method guidelines for systematic reviews in the Cochrane Back Review Group. Spine (Phila Pa 1976). 2009;34:192941.

30 Shea BJ, Reeves BC, Wells G, Thuku M, Hamel C, Moran J, et al. AMSTAR 2: a critical appraisal tool for systematic reviews that include randomised or non-randomised studies of healthcare interventions, or both. BMJ. 2017;358:j4008.

31 Guyatt G, Oxman AD, Akl EA, Kunz R, Vist G, Brozek J, et al. GRADE guidelines: 1. Introduction-GRADE evidence profiles and summary of findings tables. J Clin Epidemiol. 2011;64:383-94.

32 Balshem $H$, Helfand $M$, Schünemann $H J$, Oxman $A D$, Kunz R, Brozek J, et al. GRADE guidelines: 3 . Rating the quality of evidence. J Clin Epidemiol. 2011;64:401-6.

33 Berlim MT, Van den Eynde F, Daskalakis ZJ. Clinically meaningful efficacy and acceptability of low-frequency repetitive transcranial magnetic stimulation (rTMS) for treating primary major depression: a meta-analysis of randomized, double-blind and sham-controlled trials. Neuropsychopharmacology. 2013;38:543-51.

34 Kisely S, Li A, Warren N, Siskind D. A systematic review and metaanalysis of deep brain stimulation for depression. Depress Anxiety. 2018;35:468-80.

35 UK ECT Review Group. Efficacy and safety of electroconvulsive therapy in depressive disorders: a systematic review and metaanalysis. Lancet. 2003;361:799-808.

36 Sonmez AI, Camsari DD, Nandakumar AL, Voort JL, Kung S, Lewis $\mathrm{CP}$, et al. Accelerated TMS for depression: a systematic review and meta-analysis. Psychiatry Res. 2019;273:770-81.

37 Razza LB, Palumbo P, Moffa AH, Carvalho AF, Solmi M, Loo CK, et al. A systematic review and meta-analysis on the effects of transcranial direct current stimulation in depressive episodes. Depress Anxiety. 2020;37:594-608.

38 Nitsche MA, Paulus W. Excitability changes induced in the human motor cortex by weak transcranial direct current stimulation. J Physiol.2000;527 Pt 3: 633-9.

39 Pascual-Leone A, Catalá MD, Pascual AP. Lateralized effect of rapidrate transcranial magnetic stimulation of the prefrontal cortex on mood. Neurology. 1996;46:499-502.

40 Sehatzadeh S, Daskalakis ZJ, Yap B, Tu HA, Palimaka S, Bowen JM, et al. Unilateral and bilateral repetitive transcranial magnetic stimulation for treatment-resistant depression: a meta-analysis of randomized controlled trials over 2 decades. J Psychiatry Neurosci. 2019;44:151-63.

41 Kedzior KK, Reitz SK. Short-term efficacy of repetitive transcranial magnetic stimulation (rTMS) in depression- reanalysis of data from meta-analyses up to 2010. BMC Psychol. 2014;2:39.

42 Brunoni AR, Valiengo L, Baccaro A, Zanão TA, de Oliveira JF, Goulart A, et al. The sertraline vs. electrical current therapy for treating depression clinical study: results from a factorial, randomized, controlled trial. JAMA Psychiatry. 2013;70:383-91.

43 Sampaio-Junior B, Tortella G, Borrione L, Moffa AH, Machado-Vieira $R$, Cretaz E, et al. Efficacy and safety of transcranial direct current stimulation as an add-on treatment for bipolar depression: a randomized clinical trial. JAMA Psychiatry. 2018;75:158-66.

44 McLaren ME, Nissim NR, Woods AJ. The effects of medication use in transcranial direct current stimulation: a brief review. Brain Stimul. 2018;11:52-8.

45 Moffa AH, Martin D, Alonzo A, Bennabi D, Blumberger DM, Benseñor IM, et al. Efficacy and acceptability of transcranial direct current stimulation (tDCS) for major depressive disorder: an individual patient data meta-analysis. Prog Neuropsychopharmacol Biol Psychiatry. 2020;99:109836.

46 Bennabi D, Haffen E. Transcranial direct current stimulation (tDCS): a promising treatment for major depressive disorder? Brain Sci. 2018;8:81. 
47 Lefaucheur JP, Antal A, Ayache SS, Benninger DH, Brunelin J, Cogiamanian $\mathrm{F}$, et al. Evidence-based guidelines on the therapeutic use of transcranial direct current stimulation (tDCS). Clin Neurophysiol. 2017;128:56-92.

48 Alonzo A, Fong J, Ball N, Martin D, Chand N, Loo C. Pilot trial of home-administered transcranial direct current stimulation for the treatment of depression. J Affect Disord. 2019;252:475-83.

49 Borrione L, Moffa AH, Martin D, Loo CK, Brunoni AR. Transcranial direct current stimulation in the acute depressive episode: a systematic review of current knowledge. J ECT. 2018;34:153-63.

50 Sathappan AV, Luber BM, Lisanby SH. The dynamic duo: combining noninvasive brain stimulation with cognitive interventions. Prog Neuropsychopharmacol Biol Psychiatry. 2019;89:347-60.

51 Mutz J, Edgcumbe DR, Brunoni AR, Fu CH. Efficacy and acceptability of non-invasive brain stimulation for the treatment of adult unipolar and bipolar depression: a systematic review and metaanalysis of randomised sham-controlled trials. Neurosci Biobehav Rev. 2018;92:291-303.

52 Rossini D, Lucca A, Magri L, Malaguti A, Smeraldi E, Colombo C, et al. A symptom-specific analysis of the effect of high-frequency left or low frequency right transcranial magnetic stimulation over the dorsolateral prefrontal cortex in major depression. Neuropsychobiology. 2010;62:91-7.

53 Chen JJ, Zhao LB, Liu YY, Fan SH, Xie P. Comparative efficacy and acceptability of electroconvulsive therapy versus repetitive transcranial magnetic stimulation for major depression: a systematic review and multiple-treatments meta-analysis. Behav Brain Res. 2017;320:30-6.

54 McGirr A, Berlim MT, Bond DJ, Chan PY, Yatham LN, Lam RW. Adjunctive ketamine in electroconvulsive therapy: updated systematic review and meta-analysis. Br J Psychiatry. 2017;210:403-7.

55 Kolshus E, Jelovac A, McLoughlin DM. Bitemporal v. high-dose right unilateral electroconvulsive therapy for depression: a systematic review and meta-analysis of randomized controlled trials. Psychol Med. 2017;47:518-30.

56 Pagnin D, de Queiroz V, Pini S, Cassano GB. Efficacy of ECT in depression: a meta-analytic review. J ETC. 2004;20:13-20.

57 Kellner $\mathrm{CH}$. Brain stimulation in psychiatry: ECT, DBS, TMS and other modalities. Cambridge: Cambridge University; 2012.

58 Hersh JK. Electroconvulsive therapy (ECT) from the patient's perspective. J Med Ethics. 2013;39:171-2.

$59 \mathrm{Lin} \mathrm{CH}$, Huang CJ, Chen CC. ECT has greater efficacy than fluoxetine in alleviating the burden of illness for patients with major depressive disorder: a Taiwanese pooled analysis. Int J Neuropsychopharmacol. 2018;21:63-72.

60 Mayor S. ECT may be better than drugs for short term depression. BMJ. 2003;326:569.

61 Nuttin B, Cosyns P, Demeulemeester H, Gybels J, Meyerson B. Electrical stimulation in anterior limbs of internal capsules in patients with obsessive-compulsive disorder. Lancet. 1999;354:1526.

62 Delaloye S, Holtzheimer PE. Deep brain stimulation in the treatment of depression. Dialogues Clin Neurosci. 2014;16:83-91.

63 Mayberg HS, Lozano AM, Voon V, McNeely HE, Seminowicz D, Hamani C, et al. Deep brain stimulation for treatment-resistant depression. Neuron. 2005;45:651-60.

64 Lozano AM, Mayberg HS, Giacobbe P, Hamani C, Craddock RC, Kennedy $\mathrm{SH}$. Subcallosal cingulate gyrus deep brain stimulation for treatment-resistant depression. Biol Psychiatry. 2008;64:461-7.
65 Bergfeld IO, Mantione M, Hoogendoorn ML, Ruhé HG, Notten P, van Laarhoven J, et al. Deep brain stimulation of the ventral anterior limb of the internal capsule for treatment-resistant depression: a randomized clinical trial. JAMA Psychiatry. 2016;73:456-64.

66 Schlaepfer TE, Bewernick BH, Braeuer A, Coenen VA. A shamcontrolled study of deep brain stimulation to the medial forebrain bundle for treatment-resistant depression. Biol Psychiatry. 2016;79: 1S-438S.

67 Kennedy SH, Giacobbe P, Rizvi SJ, Placenza FM, Nishikawa Y, Mayberg HS, et al. Deep brain stimulation for treatment-resistant depression: follow-up after 3 to 6 years. Am J Psychiatry. 2011;168: 502-10.

68 Levkovitz Y, Isserles M, Padberg F, Lisanby SH, Bystritsky A, Xia G, et al. Efficacy and safety of deep transcranial magnetic stimulation for major depression: a prospective multicenter randomized controlled trial. World Psychiatry. 2015;14:64-73.

69 Tavares DF, Myczkowski ML, Alberto RL, Valiengo L, Rios RM, Gordon P, et al. Treatment of bipolar depression with deep TMS: results from a double-blind, randomized, parallel group, shamcontrolled clinical trial. Neuropsychopharmacology. 2017;42:2593601.

70 Duprat R, Desmyter S, Rudi DR, van Heeringen K, Van den Abbeele $D$, Tandt $H$, et al. Accelerated intermittent theta burst stimulation treatment in medication-resistant major depression: a fast road to remission? J Affect Disord. 2016;200:6-14.

$71 \mathrm{Li} \mathrm{CT}$, Chen MH, Juan $\mathrm{CH}$, Huang HH, Chen LF, Hsieh JC, et al. Efficacy of prefrontal theta-burst stimulation in refractory depression: a randomized sham-controlled study. Brain. 2014;137:2088-98.

72 Chung SW, Hoy KE, Fitzgerald PB. Theta-burst stimulation: a new form of TMS treatment for depression? Depress Anxiety. 2015;32: 182-92.

73 Aparício LV, Guarienti F, Razza LB, Carvalho AF, Fregni F, Brunoni AR. A systematic review on the acceptability and tolerability of transcranial direct current stimulation treatment in neuropsychiatry trials. Brain Stimul. 2016:9:671-81.

74 Loo CK, Mitchell PB, McFarquhar TF, Malhi GS, Sachdev PS. A sham-controlled trial of the efficacy and safety of twice-daily rTMS in major depression. Psychol Med. 2007;37:341-9.

75 Bikson M, Grossman P, Thomas C, Zannou AL, Jiang J, Adnan T, et al. Safety of transcranial direct current stimulation: evidence based update 2016. Brain Stimul. 2016;9:641-61.

76 Antal A, Alekseichuk I, Bikson M, Brockmöller J, Brunoni AR, Chen R et al. Low intensity transcranial electric stimulation: safety, ethical, legal regulatory and application guidelines. Clin Neurophysiol. 2017; 128:1774-809.

77 Lerner AJ, Wassermann EM, Tamir DI. Seizures from transcranial magnetic stimulation 2012-2016: results of a survey of active laboratories and clinics. Clin Neurophysiol. 2019;130:1409-16.

78 Kadiyala PK, Kadiyala LD. Anaesthesia for electroconvulsive therapy: an overview with an update on its role in potentiating electroconvulsive therapy. Indian J Anaesth. 2017;61:373-80.

79 Kalisova L, Kubinova M, Michalec J, Albrecht J, Madlova K, Raboch J. Cognitive functioning in patients treated with electroconvulsive therapy. Neuropsychiatr Dis Treat. 2018;14:3025-31.

80 Bogod NM, Sinden M, Woo C, Defreitas VG, Torres IJ, Howard AK, et al. Long-term neuropsychological safety of subgenual cingulate gyrus deep brain stimulation for treatment-resistant depression. J Neuropsychiatry Clin Neurosci. 2014;26:126-33. 